\title{
Predicting pericardial effusions in end-stage renal disease: Does pulmonary hypertension play a role?
}

\author{
De Cicco Ignacio A ${ }^{1}$, McBride Cameron ${ }^{1}$, Llanos-Chea Fiorella ${ }^{1}$, Gacad Vincent $E^{1}$, Yu Patrick L ${ }^{1}$, Benjamin-Garner Ruby A $^{2}$ and Aisenberg
} Gabriel M ${ }^{1 *}$

${ }^{1}$ Division of Internal Medicine, University of Texas McGovern School of Medicine, USA

${ }^{2}$ Division of Clinical Research, University of Texas, McGovern School of Medicine, USA

\begin{abstract} $\mathrm{mmHg}$ and the presence of pericardial effusion.

\section{Introduction}

Pericardial effusion affects as many as $19-35 \%$ of patients with end-stage renal disease (ESRD) [1-6]. Though ESRD is the presumed cause of up to $12 \%$ of pericardial effusions categorized as small or larger than small, the pathophysiology is incompletely understood $[7,8]$. Although the prevalence of pericardial effusion has declined in the face of more efficient hemodialysis, it's unclear whether this is mainly due to removal of metabolic wastes or optimization of volume status and hemodynamics [9-11].
\end{abstract}

Background: Pericardial effusion occurs, in variable extent, in 35\% of patients on hemodialysis. Pulmonary hypertension is considered responsible for the generation and maintenance of pericardial effusion, but such association has not been proven in hemodialysis patients.

Methods: We retrospectively collected data on patients with end-stage renal disease (ESRD) on hemodialysis who had two or more transthoracic echocardiograms. We investigated demographic and echocardiographic data focused on trying to find an association between right ventricular systolic pressure (RVSP) above 40

Results: 129 patients had 395 echocardiograms. Sixty-six percent of studies showed RVSP above $40 \mathrm{~mm} \mathrm{Hg}$, while $43 \%$ showed variable degrees of pericardial effusion. We found a weak positive correlation between these variables ( $\mathrm{R}$ coefficient $=0.16$ ). RVSP above $40 \mathrm{mmHg}$ predicted the presence (but not severity) of pericardial effusion even in patients without severe systolic heart failure. In follow-up TTE, the incidence of a new effusion was higher among patients whose RVSP was or rose above $40 \mathrm{~mm} \mathrm{Hg}$ compared to those with normal RVSP (20\% vs. $7 \%, P=0.03$ ). Hemodialysis schedule did not modify the results.

Conclusions: A single RVSP above $40 \mathrm{mmHg}$ predicts the presence but not severity of pericardial effusion in ESRD patients, while consistently elevated RVSP appears to also predict the emergence of new, mostly mild pericardial effusion among those patients.

Pulmonary hypertension ( $\mathrm{PH})$ has been described as a possible cause of pericardial effusion, and it may represent a necessary condition for the development and maintenance of pericardial effusion [12-14]. However, this issue has not been addressed among patients with ESRD. We aim to assess whether pulmonary hypertension measured by noninvasive means predicts the presence and persistence of pericardial effusions in a cohort of ESRD patients.

\section{Methods}

The study was undertaken at Lyndon B. Johnson Hospital, a tertiary care center in Houston, Texas, after obtaining Institutional Review Board approval (protocol HSC-MS-14-0115); patient consent requirements were waived. We retrospectively collected data on patients with ESRD on hemodialysis who had a minimum of two fully descriptive transthoracic echocardiograms (TTE) performed in our hospital between January 2010 and February 2016. From the electronic medical records, we reviewed demographic data (gender, age), if the patients were receiving scheduled (SHD) or emergent dialysis (EHD), and reports of their TTE. From the latter we extracted information on left ventricular ejection fraction (EF) and right ventricular systolic pressure (RVSP), as well as the presence and severity of pericardial effusion (mild, moderate, severe). RVSP was estimated considering the jet of tricuspid regurgitation by Doppler, compared to a constant value of right atrial pressure as recommended in the literature. Being that RVSP presents as a range of values in the normal population, for our cohort we used the mean value of $40 \mathrm{~mm} \mathrm{Hg}$, above which we defined it as being elevated [15] Patients with fewer than 2 TTE or those on whom the RVSP could not be measured or was not reported were excluded.

\section{Statistical methods}

Categorical variables were analyzed using the Fisher exact test, and discrete variables were analyzed using the Student's $t$ test for unpaired samples. A two-sided $P<0.05$ was considered indicative of statistical significance. A correlation coefficient between values of RVSP and the presence of pericardial effusion was calculated. We used SPSS 23 software.

Correspondence to: Aisenberg, Gabriel M, Division of Internal Medicine, University of Texas Mc Govern School of Medicine, 6431 Fannin street, MSB 1.122, Houston, TX 77030, Tel: 713-500-6714; Fax:713-500-6722; E-mail: Gabriel.M.Aisenberg@uth.tmc.edu

Key words: End-stage renal disease, pulmonary hypertension, pericardial effusion, hemodialysis

Received: February 13, 2018; Accepted: February 23, 2018; Published: February 28,2018 


\section{Results}

Baseline characteristics for our cohort can be found on table 1 . The patients on SHD were dialyzed three times a week on average, while patients on EHD received dialysis every $7 \pm 50$ days. The time elapsed between the first and last TTE was 526 days (range 14 to 1862 days).

The presence of pericardial effusion was more common among those with elevated RVSP $\{139$ of 295 (47\%), vs. 49 of $136(36 \%)$; $P=0.001\}$. Moreover, there was a weak positive correlation between the value of RVSP and the presence of pericardial effusion $(R=0.17)$. Considering that heart failure with low ejection fraction is a common comorbid condition among patients with end-stage renal disease, also known to cause class 2 pulmonary hypertension [16], we confirmed the correlation between elevated RVSP and the presence of pericardial effusion after excluding 65 patients with left-ventricular ejection fraction lower than $35 \%\{111$ of 207 (54\%) vs. 42 of 123 (34\%); $P<0.001\}$.

Most patients had mild pericardial effusion. Of the 9 patients with severe pericardial effusion, $6(1.5 \%)$ had RVSP $>40 \mathrm{~mm} \mathrm{Hg}$ and $3(0.7 \%$, $P=1.00$ ) did not.

The incidence of new effusion in a follow-up TTE was more frequent among patients whose RVSP was or rose above $40 \mathrm{~mm} \mathrm{Hg}$ compared to those with normal RVSP ( $20 \%$ vs. $7 \%, P=0.03)$. However, effusion did not decrease differently when RVSP stayed or decreased under $40 \mathrm{~mm} \mathrm{Hg}$, compared to values above that number (19\% vs. $13 \%$, $P=0.33$ ).

The frequency of hemodialysis (SHD versus EHD) did not modify the influence of RVSP above $40 \mathrm{~mm} \mathrm{Hg}$ on the presence of pericardial effusion.

\section{Discussion}

We have previously published our experience with 251 ESRD patients at a county hospital in our institution. In that cohort, 89 of 251 (35\%) patients admitted with previously diagnosed ESRD had varying degrees of pericardial effusion [17]. In that series, patients with pericardial effusion were more likely to have left ventricular systolic dysfunction, which raised the suspicion that group-2 pulmonary hypertension (secondary to left atrial or ventricular pressure) may have been responsible for these effusions [18]. The present study seems to support these findings, but also proves an association of elevated RVSP and pericardial effusion even among patients whose left ventricular ejection fraction is not severely impaired. Interestingly, this association is not present when analyzing the few patients with severe pericardial effusion.

Table 1. Baseline characteristics of the study population ( $\mathrm{n}=129)$.

\begin{tabular}{|l|l|}
\hline Age & $\mathbf{4 9} \pm \mathbf{1 5}$ years \\
\hline Gender (male) & $62(48 \%)$ \\
\hline Type of hemodialysis & \\
\hline Scheduled (3 times per week) & $65(50 \%)$ \\
\hline Emergent & $64(50 \%)$ \\
\hline Number of analyzed echocardiograms & 395 \\
\hline First & $129(100 \%)$ \\
\hline Second & $129(100 \%)$ \\
\hline Third & $76(59 \%)$ \\
\hline Fourth & $42(33 \%)$ \\
\hline Fifth & $19(15 \%)$ \\
\hline RVSP $>40 \mathrm{~mm} \mathrm{Hg}$ & $259(66 \%)$ \\
\hline Pericardial effusion & $169(43 \%)$ \\
\hline Mild & $140(83 \%)$ \\
\hline Moderate & $20(12 \%)$ \\
\hline Severe & $9(5 \%)$ \\
\hline
\end{tabular}

Past studies have tried to explain whether uremic pericarditis or volume abnormalities are more often leading to the presence of effusion. In one study, of the 150 patients who had an echocardiogram prior to undergoing a dialysis session, 93 of them (62\%) had some degree of pericardial effusion [19]. Although the first three months of hemodialysis decreased the incidence of pericardial effusion in this cohort, that change did not correlate with the degree of metabolic correction, thus arguing that optimizing volume status may be the most critical factor in prevention of pericardial effusion in these patients. In our patient population, dialysis schedule did not modify the risk for pericardial effusion, even when adjusted for RVSP value.

It is noteworthy that most cases of pericardial effusion were mild. Therefore, even when understanding the underlying pathophysiology, it seems that any intervention to manage $\mathrm{PH}$ in this setting may not be indicated.

The main limitation of our study is its retrospective nature, preventing the investigators from knowing the indication for the TTE among the study patients, and therefore, from instructing the cardiologist on the relevance of acquiring data on $\mathrm{PH}$. Moreover, we used a single echocardiographic variable as a surrogate for PH (RVSP), while the prospective evaluation of other described variables might have modified our results [15].

\section{Conclusion}

A single RVSP above $40 \mathrm{~mm} \mathrm{Hg}$ predicts coexisting effusion, even when excluding patients with severe systolic heart failure. Rising RVSP in subsequent echocardiograms was associated with increased incidence of pericardial effusion, but decreasing values was not associated with lower incidence of such finding. Pulmonary hypertension may have a causative role in the presence but not severity of pericardial effusion among patients with end-stage renal disease. Most cases of pericardial effusion in our cohort were mild, creating no need for change in the therapy of pulmonary hypertension. Scheduled dialysis did not appear to prevent pericardial effusion better than emergent hemodialysis in our cohort.

\section{References}

1. J H Kleiman, J Motta, E London, J P Pennell, R L Popp (1978) Pericardial effusions in patients with end-stage renal disease. Br Heart J 40:190-193. [Crossref]

2. Drueke T, LePailleur C, Zingraff F (1980) Uremic cardiomyopathy and pericarditis Adv Nephrol Necker Hosp 9: 33-70. [Crossref]

3. Comty CM, Cohen SL, Shapiro FL (1971) Pericarditis in chronic uremia and its sequels. Ann Intern Med 75:173-183. [Crossref]

4. Rostand SG, Rutsky EA (1990) Pericarditis in end-stage renal disease. Cardiol Clin 8: 701-717.

5. Rutsky EA, Rostand SG (1987) Treatment of uremic pericarditis and pericardial effusion. Am J Kidney Dis 10: 2-8. [Crossref]

6. Rutsky EA, Rostand SG (1989) Pericarditis in end-stage renal disease: clinical manifestations and management. Semin Dial 2: 25-30.

7. Tsang TSM, Sinak LJ, Oh JK (2009) Pericardial Effusion, Tamponade, and Constriction. In Nihoyannopoulos P and Kisslo J editors. Echocardiography. Springer-Verlag London Limited 297-310.

8. Sagristà-Sauleda J, Mercé J, Permanyer-Miralda G, Soler-Soler J (2000) Clinical clues to the causes of large pericardial effusions. Am J Med 109: 95-101. [Crossref]

9. Corey GR, Campbell PT, Van Trigt P, Kenney RT, O'Connor CM, et al. (1993) Etiology of large pericardial effusion. Am J Med 95: 209. [Crossref]

10. Silverberg S, Oreopoulos DG, Wise DJ, Uden DE, Meidok H, et al. (1977) Pericarditis in patients undergoing long term hemodialysis and peritoneal dialysis Incidence, complications and management. Am J Med; 63: 874-880. [Crossref] 
11. Sadjadi SA, Mashahdian A (2015) Uremic pericarditis: a report of 30 cases and review of the literature. Am J Case Rep 16:169-73. [Crossref]

12. Grapsa J, Pereira Nunes MC, Tan TC, Cabrita IZ, Coulter T, et al. (2015) Echocardiographic and Hemodynamic Predictors of Survival in Precapillary Pulmonary Hypertension: Seven Year Follow-Up. Circ Cardiovasc Imaging 8: 45-54. [Crossref]

13. Raymond RJ, Hinderliter AL, Willis PW, Ralph D, Caldwell EJ, et al. (2002) Echocardiographic Predictors of Adverse Outcomes in Primary Pulmonary Hypertension. J Am Coll Cardiol 39:1214-1219. [Crossref]

14. Mellins RB, Levine R, Fishman AP (1970) Effect of systemic and pulmonary venous hypertension on pleural and pericardial fluid accumulation. J Appl Physiol 29:564-569. [Crossref]
15. Galie N, Humbert M, Vachiery JL (2016) 2015 ESC/ERS Guidelines for the diagnosis and treatment of pulmonary hypertension. Eur Heart J 37: 67-119.

16. Simonneau G, Gatzoulis MA, Adatia I, Celermajer D, Denton C, et al. (2013) Updated clinical classification of pulmonary hypertension. JACC 62: D34-41. [Crossref]

17. Chang KW, Aisenberg GM (2015) Pericardial effusion in Patients with End-Stage Renal Disease. Tex Heart Inst $J$ 42: 596. [Crossref]

18. Farber HW, Loscalzo J (2004) Pulmonary arterial hypertension. $N$ Engl J Med 351: 1655-1665. [Crossref]

19. Yoshida K, Shiina A, Asano Y, Hosoda S (1980) Uremic pericardial effusion: detection and evaluation of uremic pericardial effusion by echocardiography. Clin Nephrol 13 260-268. [Crossref]

Copyright: $@ 2018$ De Cicco IA. This is an open-access article distributed under the terms of the Creative Commons Attribution License, which permits unrestricted use, distribution, and reproduction in any medium, provided the original author and source are credited. 